\title{
EFEITO DA TEMPERATURA SOBRE O PERFIL DE METABÓLITOS DO SUCO DE MARACUJÁ (PASSIFLORA EDULIS)
}

\author{
M. V. L. SOARES ${ }^{1}$, C. G. de SOUZA ${ }^{1}$, N.J. WURLITZER ${ }^{2}$, RIBEIRO, P.R.V ${ }^{2}$ e E. S. de \\ BRITO $^{3}$ \\ ${ }^{1}$ Universidade Federal do Ceará, Departamento de Engenharia Química \\ ${ }^{2}$ Embrapa Agroindústria Tropical - CNPAT, Rua Doutora Sara Mesquita, 2270 - Bairro \\ Pici, 60511-110 Fortaleza, Ceará, Brasil \\ ${ }^{3}$ Tropical Agribusiness Embrapa. \\ E-mail para contato: marcinha_lacerd@yahoo.com.br
}

\begin{abstract}
RESUMO - As frutas, principais fontes dietéticas de polifenóis, podem perder alguns nutrientes com o processamento térmico, em função de fatores intrínsecos e extrínsecos. Atualmente, o interesse pelas frutas e seus derivados vem aumentando em decorrência de suas propriedades funcionais. No entanto, os efeitos do processo sobre estes compostos ainda são pouco conhecidos. Neste estudo, objetivou-se avaliar os compostos presentes em suco de maracujá após o tratamento térmico de pasteurização e de esterilização em diferentes condições de tempo e temperatura $\left(85^{\circ} \mathrm{C}\right.$ e $140^{\circ} \mathrm{C}$ por 15,30 e 60 segundos). Os maracujás foram adquiridos e tratados termicamente; para extração dos polifenóis, utilizou-se metanol e as amostras foram analisadas em LC-MS. Os resultados foram comparados a uma amostra controle (sem tratamento), em que foram encontrados 27 picos, dos quais 4 foram degradados, 7 foram formados e 16 permaneceram inalterados. Com o aumento da temperatura houve degradação de compostos fenólicos. O processo térmico pode ser controlado a partir de dados obtidos sobre o perfil de suco de maracujá submetido a tratamento térmico.
\end{abstract}

\section{INTRODUÇÃO}

As frutas em geral são fontes dietéticas de polifenóis, e vários estudos indicam presença dessa substância em maracujá (Zeraik e Yariwake, 2010), podendo assim indicar o potencial dessa fruta como alimento funcional.

O maracujá amarelo (Passiflora edulis ssp.) é uma fruta tropical, que possui sabor bastante atrativo. Esta fruta é largamente utilizada em sorvetes, mousses e principalmente em suco. O suco de maracujá é promissor no crescimento da comercialização e consumo de sucos prontos para beber, porque o maracujá é uma fruta típica do Brasil, disponível em abundância ao longo do ano (Saron et al, 2007). Nos últimos anos, o mercado brasileiro de sucos prontos vem crescido bastante na categoria das bebidas não-alcóolicas, e existe previsão de que as exportações de sucos de frutas devam dobrar nos próximos anos (Labruna, 2001; Haberli, 2003;).

Atualmente, tem-se atribuído aos alimentos, não só as funções de nutrição e de fornecer apelo sensorial, mas também uma função relacionada à resposta físiológica específica produzida por alguns alimentos, os quais são chamados de alimentos funcionais. 
Estes alimentos podem prevenir, curar ou auxiliar na recuperação de determinadas doenças (Culhane, 1995).

Com o processamento do suco, o perfil de metabólitos poderá variar devido a fatores intrínsecos e extrínsecos. Porém, estes efeitos ainda são pouco conhecidos. No presente trabalho, objetivou-se avaliar os compostos presentes em suco de maracujá após o tratamento térmico de pasteurização e de esterilização em diferentes condições de tempo e temperatura $\left(85^{\circ} \mathrm{C}\right.$ e $140^{\circ} \mathrm{C}$ por 15,30 e 60 segundos $)$.

\section{MATERIAIS E MÉTODOS}

\subsection{Obtenção das amostras}

Os frutos de Passiflora edulis ssp., foram adquiridos na Central de Abastecimento S/A de Fortaleza - CEASA-CE. Foi feita a higienização dos frutos com água e sabão e depois a sanitização em água clorada $(150 \mathrm{mg} / \mathrm{L})$. Posteriormente a polpa foi obtida.

\subsection{Tratamento térmico}

O suco foi submetido a tratamento térmico de pasteurização e esterilização em diferentes condições de tempo e temperatura $\left(85^{\circ} \mathrm{C}\right.$ e $140^{\circ} \mathrm{C}$ por 15,30 e 60 segundos $)$. Foi utilizado o trocador de calor tubular da marca Armfield (FT 74 UHT/HTST).

\subsection{Determinações analíticas}

Após a extração com metanol, foi feita a identificação dos extratos por cromatografia líquida e espectrometria de massa no equipamento UPLC (Aquity) e QToF (Xevo), no modo negativo. Foi utilizada a coluna analítica Acquity UOLC BEH C18 $(1,7 \mu \mathrm{m}, 2.1 \mathrm{~mm} \times$ $150 \mathrm{~mm}$ ) com fluxo de $0,5 \mathrm{~mL} / \mathrm{min}$, volume de injeção de $20 \mu \mathrm{L}$ e o tempo total da corrida foi de 15 minutos. A fase móvel utilizada foi água / ácido fórmico $0,1 \%$ e acetonitrila / ácido fórmico $0,1 \%(\mathrm{v} / \mathrm{v})$.

\section{RESULTADOS E DISCUSSÃO}

Os resultados obtidos foram comparados a uma amostra controle (sem tratamento térmico), em que foram encontrados 27 picos, dos quais 4 foram degradados, 7 foram formados e 16 permaneceram inalterados. Mostrando assim a influência ocasionada nos compostos pela temperatura, indicando que alguns compostos foram formados por tornaremse biodisponíveis, enquanto que outros sofreram influência negativa da temperatura.

Nas figuras 1,2 e 3 são mostrados cromatogramas representativos da amostra controle, e das duas temperaturas utilizadas $\left(85 \mathrm{e} 140^{\circ} \mathrm{C}\right)$. Os cromatogramas apresentaram perfis semelhantes, porém quando se faz um estudo detalhado de cada tempo de retenção, alguns valores são alterados. 


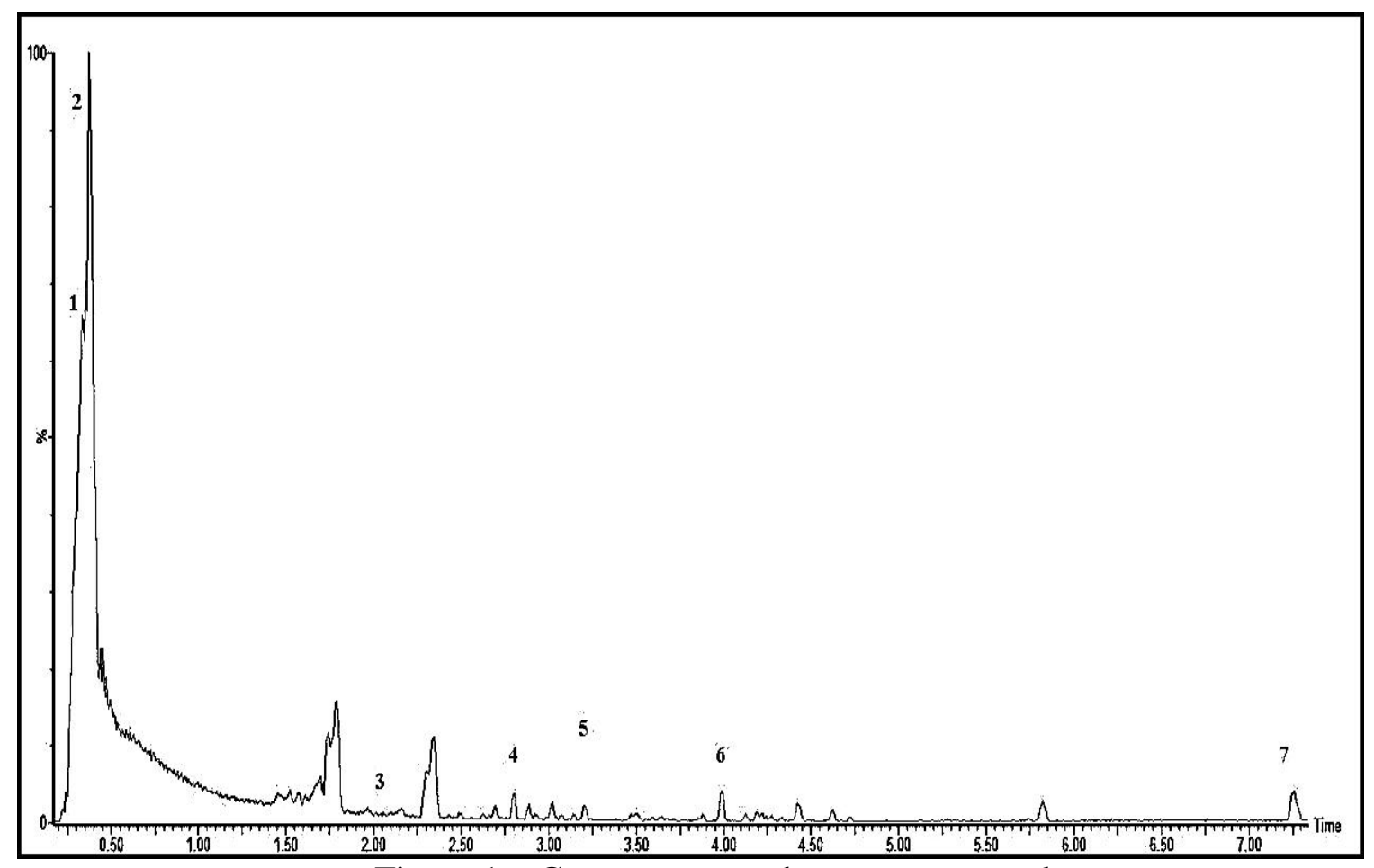

Figura 1 - Cromatograma da amostra controle.

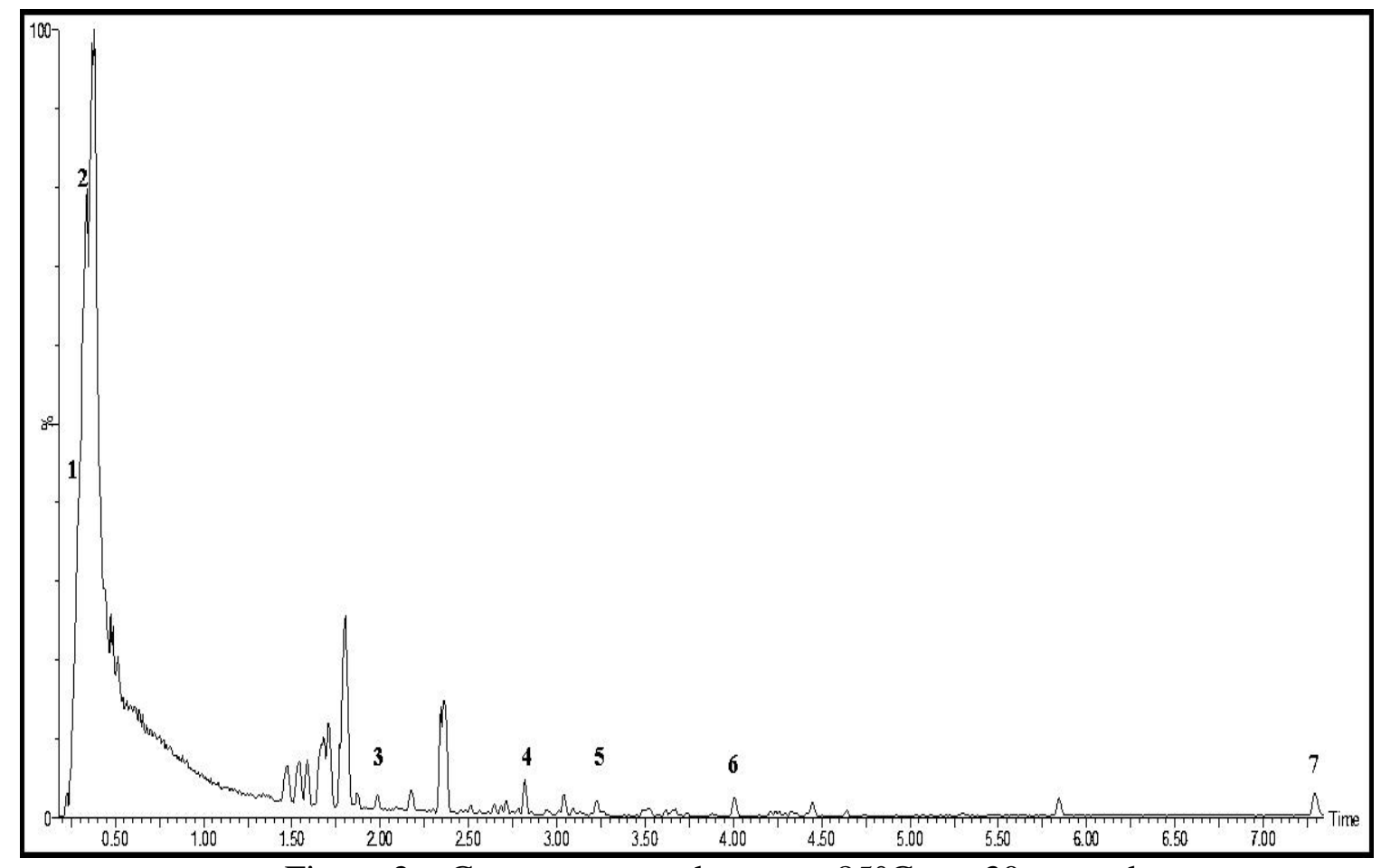

Figura 2 - Cromatograma do suco a $85^{\circ} \mathrm{C}$ por 30 segundos. 


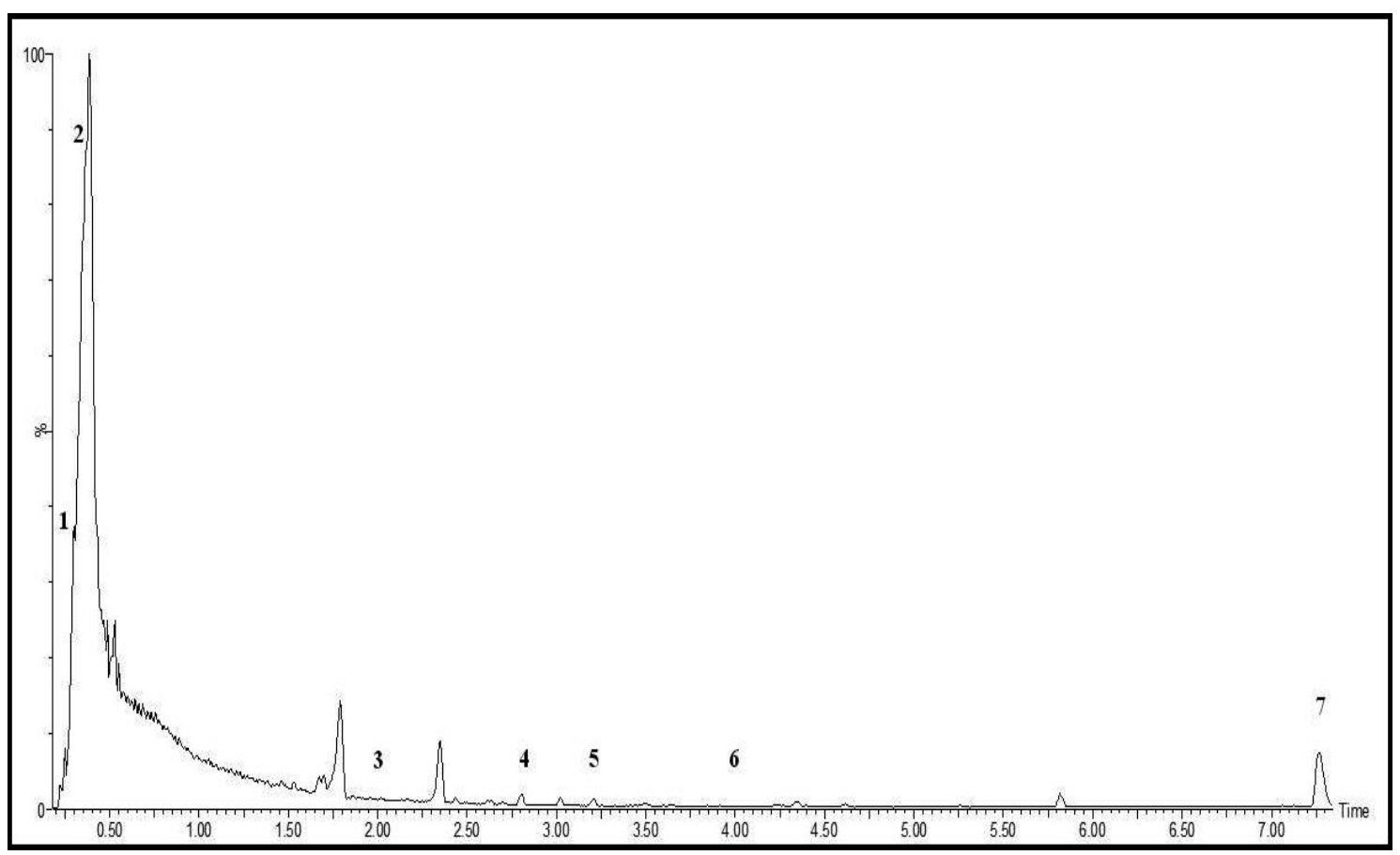

Figura 3. Cromatograma do suco a $140^{\circ} \mathrm{C}$ por 30 segundos.

Na Tabela 1 estão especificados 7 picos cromatográficos relevantes (destacados nas Figuras 1,2 e 3), que apareceram com maior frequência nos cromatogramas. Os compostos foram reconhecidos a partir de dados das massas moleculares obtidos na análise de LC-MS e subsequente confirmação por comparação com dados da literatura (Tabela 1).

Tabela 1. Picos cromatográficos relevantes

\begin{tabular}{|c|c|c|c|c|c|c|}
\hline Pico & $\begin{array}{c}\text { Tempo } \\
\text { retenção } \\
(\text { min) }\end{array}$ & $\begin{array}{l}\text { Fórmula } \\
\text { Molecular }\end{array}$ & $\begin{array}{c}{[\mathrm{M}-\mathrm{H}]^{-}} \\
\text {Experimental }\end{array}$ & $\begin{array}{l}{[\mathrm{M}-\mathrm{H}]^{-}} \\
\text {Literatura }\end{array}$ & Substância & $\begin{array}{l}\text { Referência } \\
\text { Bibliográfica }\end{array}$ \\
\hline 1 & 0,25 & $\mathrm{C}_{9} \mathrm{H}_{8} \mathrm{O}_{4}$ & 179,054 & 179,0349 & Ácido cafeico & $\begin{array}{c}\text { Vallverdu- } \\
\text { Queralt et al., } \\
\text { 2012) }\end{array}$ \\
\hline 2 & 0,37 & $\mathrm{C}_{7} \mathrm{H}_{12} \mathrm{O}_{6}$ & 191,0280 & 191,0556 & Ácido quínico & $\begin{array}{c}\text { Vallverdu- } \\
\text { Queralt et al., } \\
\text { 2012) }\end{array}$ \\
\hline 3 & 2,17 & $\mathrm{C}_{15} \mathrm{H}_{10} \mathrm{O}_{5}$ & 269,10 & 269,337 & Apigenina & $\begin{array}{c}\text { Vallverdu- } \\
\text { Queralt et al., } \\
\text { 2012) }\end{array}$ \\
\hline 4 & 2,8 & & 455,230 & 455,000 & Digalloylpentose & $\begin{array}{c}\text { (Gordon et al., } \\
\text { 2011) }\end{array}$ \\
\hline 5 & 3,2 & $\mathrm{C}_{9} \mathrm{H}_{10} \mathrm{O}_{5}$ & 199,106 & 199,0606 & Ácido siríngico & $\begin{array}{l}\text { Vallverdu- } \\
\text { Queralt et al., } \\
\text { 2012) }\end{array}$ \\
\hline 6 & 3,99 & & 507,23 & 507,000 & Ácido vanoleico & $\begin{array}{c}\text { (Fernandes } e t \\
\text { al., } 2011\end{array}$ \\
\hline 7 & 7,26 & $\mathrm{C}_{27} \mathrm{H}_{30} \mathrm{O}_{15}$ & 593,261 & 593,000 & Vicenina-2 & $\begin{array}{c}\text { (Zucolotto et al., } \\
\text { 2012) }\end{array}$ \\
\hline
\end{tabular}


A Tabela 2 mostra os valores das substâncias encontradas na amostra controle e nas diferentes condições de tempo e temperatura.

Tabela 2- Compostos encontrados nos cromatogramas

\begin{tabular}{|c|c|c|c|c|c|c|c|c|}
\hline Tratamento & $\begin{array}{c}\text { Sem } \\
\text { tratamento }\end{array}$ & $\begin{array}{l}85^{\circ} \mathrm{C} / \\
15 \mathrm{seg}\end{array}$ & $\begin{array}{l}85^{\circ} \mathrm{Cl} \\
\text { 30seg }\end{array}$ & $\begin{array}{l}85^{\circ} \mathrm{C} / \\
60 \text { seg }\end{array}$ & $\begin{array}{c}140^{\circ} \mathrm{C} / \\
15 \mathrm{seg}\end{array}$ & $\begin{array}{c}140^{\circ} \mathrm{Cl} \\
30 \mathrm{seg}\end{array}$ & $\begin{array}{c}140^{\circ} \mathrm{C} / \\
60 \mathrm{seg}\end{array}$ & \\
\hline $\begin{array}{l}\text { Tempo } \\
\text { retenção } \\
(\text { min) }\end{array}$ & $(\mathbf{M}-\mathbf{H})^{-}$ & $(\mathbf{M}-\mathbf{H})^{-}$ & $(\mathbf{M}-\mathrm{H})^{-}$ & $(\mathbf{M}-\mathbf{H})^{-}$ & $(\mathbf{M}-\mathrm{H})^{-}$ & $(\mathbf{M}-\mathbf{H})^{-}$ & $(\mathbf{M}-\mathrm{H})^{-}$ & Substância \\
\hline 0,25 & 179,053 & 179,12 & 179,07 & 179,13 & 179,05 & 179,054 & 179,05 & Ácido cafeico \\
\hline 0,37 & 191,0168 & 191,03 & 191,03 & 191,01 & 191,02 & 191,021 & 191,02 & Ácido quinico \\
\hline 1,61 & 340,0981 & 439,19 & 0 & 0 & 0 & 0 & 0 & Desconhecida \\
\hline 1,66 & 461,1587 & 340,1 & 341,13 & 340,11 & 340,11 & 340,098 & 340,1 & Desconhecida \\
\hline 1,77 & 486,144 & 0 & 0 & 0 & 0 & 0 & 0 & Desconhecida \\
\hline 1,8 & 0 & 486,16 & 486,2 & 486,19 & 486,15 & 486,147 & 486,15 & Desconhecida \\
\hline 1,96 & 361,1504 & 361,16 & 361,18 & 361,17 & 361,16 & 361,149 & 361,15 & Desconhecida \\
\hline 2,17 & 0 & 0 & 268,11 & 269,12 & 269,11 & 0 & 0 & Apigenina \\
\hline 2,33 & 761,174 & 761,26 & 761,22 & 761,21 & 761,19 & 761,125 & 761,19 & Desconhecida \\
\hline 2,69 & 561,2444 & 0 & 0 & 0 & 0 & 0 & 0 & Desconhecida \\
\hline 2,7 & 0 & 561,27 & 561,29 & 561,28 & 561,11 & 0 & 0 & Desconhecida \\
\hline 2,8 & 455,2036 & 455,23 & 455,23 & 455,23 & 455,22 & 455,214 & 455,22 & Digalloylpentose \\
\hline 2,89 & 723,4933 & 0 & 0 & 0 & 0 & 0 & 0 & Desconhecida \\
\hline 3,02 & 517,1854 & 517,21 & 517,22 & 517,22 & 517,2 & 517,194 & 517,19 & Desconhecida \\
\hline 3,2 & 199,0973 & 199,11 & 199,11 & 199,11 & 199,1 & 199,098 & 199,1 & Ácido siríngico \\
\hline 3,99 & 507,2362 & 507,27 & 507,27 & 507,27 & 0 & 0 & 0 & Ácido valoneico \\
\hline 7,26 & 593,261 & 593,3 & 593,31 & 593,28 & 593,27 & 593,261 & 593,26 & Vicenina-2 \\
\hline
\end{tabular}

Pela tabela 2 percebe-se que o ácido cafeico, ácido quínico, Digalloylpentose, Ácido siríngico e Vicenina-2 permaneceram tanto na amostra controle como nos diferentes tratamentos térmicos. A apigenina apareceu nas seguintes condições: $85^{\circ} \mathrm{C} / 30$ e 60 seg e a $140^{\circ} \mathrm{C} / 15$ segundos. Já o ácido vanoleico desapareceu a partir de $140^{\circ} \mathrm{C} / 15$ segundos.

\section{CONCLUSÃO}

O processo térmico pode ser controlado a partir de dados obtidos sobre o perfil de suco de maracujá submetido a tratamento térmico. O processo térmico pode alterar o perfil de metabólitos, na maioria das vezes gerando a degradação de compostos. Estudos futuros deverão ser realizados a fim de diminuir a degradação dos compostos de interesse, escolhendo-se as melhores condições de tempo e temperatura. 


\section{AGRADECIMENTOS}

À Rede Passitec,pela motivação a pesquisa, à capes pela e concessão de bolsa de estudo e a EMBRAPA pelo apoio dado e pela disponibilidade de suas dependências para realização do trabalho.

\section{REFERÊNCIAS}

CULHANE, C. (1995). Nutraceuticals/Functional Foods - an exploratory survey on Canada's potential. Toronto: International Food Focus Limited.

FERNANDES, A.; SOUSA, A.; MATEUS, N.; CABRAL, M.; de FREITAS, V. Analysis of phenolic compounds in cork from Quercus súber L. by HPLC-DAD/ESI-MS. Food Chemistry (125), 2011, p. 1398-1405.

GORDON, A.; JUNGFER, E.; da SILVA, B.A.; MAIA, J.G.S.; MARX, F. Phenolic constituents and antioxidant capacity of four underutilized fruits from the amazon region. Agric. Food Chem., 2011, 59 (14), pp 7688-7699.

HABERLI, L. Sucos prontos para gerar lucro. Embalagem Marca, São Paulo v. 5, n. 45, p.16-22, maio 2003.

LABRUNA, J. C. Sucos prontos: matam a sede do mercado. Engarrafador Moderno, São Paulo, v. 10, n. 84, p. 34-38, abr., 2001.

SARON, E.S.; DANTAS, S.T.; de MENEZES, H.C.; SOARES, B.M.C.; NUNES, M.F. Estabilidade sensorial de suco de maracujá pronto para beber acondicionado em latas de aço. Ciênc. Tecnol. Aliment., Campinas, 27(4): 772-778, out.-dez. 2007.

VALLVERDU-QUERALT, A. JÁUREGUI, O.; MEDINA-REMÓN, A.ELAMUELARAVENTÓS, R.M. Evaluation of a Method To Characterize the Phenolic Profile of Organic and Conventional Tomatoes. Journal of Agricultural and Food Chemistry, v. 60, n. 13, p. 3373-3380, Apr 4 2012. ISSN 0021-8561. Disponível em: < <Go to ISI $>$ ://WOS:000302336500006 >.

ZERAIK, M.L. E YARIWAKE, J.H. Quantification of isoorientin and total flavonoids in Passiflora edulis fruit pulp by HPLC-UV/DAD. Microchem $J$, in press, doi:10.1016/j.microc.2010.02.003.

ZUCOLOTTO, S. M.; FAGUNDES, C.; REGINATTO, F.H.; RAMOS, F.A.; CASTELLANOS, L.; DUQUEB, C.; SCHENKEL, E.P. Analysis of C-glycosyl Flavonoids from South American Passiflora Species by HPLC-DAD and HPLC-MS Phytochemical Analysis Volume 23, Issue 3. Phytochemical Analysis. 23: 232-239 p. 2012. 